\title{
Dynamics model analysis of bacteriophage infection of bacteria
}

\author{
Xiaoping $\mathrm{Li}^{1}$, Rong Huang ${ }^{2}$ and Minyuan $\mathrm{He}^{1^{*}}$ (D)
}

*Correspondence: hmy0735@126.com

${ }^{1}$ College of Mathematics and Finance, Xiangnan University, Hunan, P.R. China

Full list of author information is

available at the end of the article

\section{Springer}

\begin{abstract}
A bacteriophage (in short, phage) is a virus that can infect and replicate within bacteria. Assuming that uninfected and infected bacteria are capable of reproducing with logistic law, we investigate a model of bacteriophage infection that resembles simple SI-models widely used in epidemiology. The dynamics of host-parasite co-extinctions may exhibit four scenarios: hosts and parasites go extinct, parasites go extinct, hosts go extinct, and hosts and parasites coexist. By using the Jacobian matrix and Bendixson-Dulac theory, local and global stability analysis of uninfected and infected steady states is provided; the basic reproduction number of the model is given; general results are supported by numerical simulations. We show that bacteriophages can reduce a host density. This provides a theoretical framework for studying the problem of whether phages can effectively prevent, control, and treat infectious diseases.
\end{abstract}

Keywords: Bacteriophage; Virus; Stability; Numerical simulation

\section{Introduction}

A bacteriophage (in short, phage) is a virus that can infect and replicate within bacteria. Bacteriophages were discovered by Frederick Twort in 1915 [1] and Felix d'Herelle in 1917 [2]. Bacteriophages consist of a core of genetic material (nucleic acid) surrounded by a protein capsid. Usually a phage follows one of two life cycles: lytic (virulent) or lysogenic (temperate). When lytic phages infect bacteria, they attach their tails to the bacterial surface and then inject DNA from their heads into the bacteria. The DNA that enters the bacteria, being biosynthesised of the raw material of the host bacteria, causes proliferation of new daughter phages. Reaching a certain amount, these phages give rise to bacterial cells bursting, after which they can multiply rapidly and form hundreds of daughter phage particles. Lysogenic phages incorporate their nucleic acid into the chromosome of the host cell and replicate with it as a unit without destroying the cell. Under certain conditions, lysogenic phages can be induced for which a lytic cycle takes place. Each daughter phage can infect another bacterial cell, and the process is repeated over and over again, so that the phages can kill many cells. It has long been noted that phage therapy can be used to treat pathogenic bacterial infections. In particular, phage therapy has been widely used in livestock and poultry breeding (see [3, 4]), aquaculture (see [5]), food production (see [6]), and other fields (see $[7,8]$ ). Also, phage therapy proved itself as an important tool in the

(c) The Author(s) 2021. This article is licensed under a Creative Commons Attribution 4.0 International License, which permits use sharing, adaptation, distribution and reproduction in any medium or format, as long as you give appropriate credit to the original author(s) and the source, provide a link to the Creative Commons licence, and indicate if changes were made. The images or other third party material in this article are included in the article's Creative Commons licence, unless indicated otherwise in a credit line to the material. If material is not included in the article's Creative Commons licence and your intended use is not permitted by statutory regulation or exceeds the permitted use, you will need to obtain permission directly from the copyright holder. To view a copy of this licence, visit http://creativecommons.org/licenses/by/4.0/. 
treatment of human diseases such as diarrheal diseases caused by e. coli, shigella or vibrio, and wound infections caused by skin facultative pathogens (for example, staphylococcus and streptococcus). In recent years, phage therapy has also been used for systemic and even intracellular infections. It has been 100 years since the discovery of phage, and the research on phage has never stopped. With the emergence of antibiotics, phage therapy was gradually ignored. However, the widespread antibiotic resistance of bacteria in recent years has made the research on bacteriophages a hot spot. To which extent phages can replace antibiotics as a new treatment for bacterial infections is still under debate, and probably it will take some time for them to appear in clinical trials. However, finding a theoretical basis for studying the relationship between phage and bacteria is a problem of great importance and formidable complexity. This paper attempts to model the dynamic stability of phage infected bacteria, to discuss the relationship between phage and bacteria, and to provide some theoretical basis for whether phages can effectively prevent, control, and treat infectious diseases.

Phages can be thought of as organisms that prey on bacteria, or parasites that host bacteria. To the best of our knowledge, there are many dynamics models of viral infection in host cells (see [9-20] and the references therein). For example, in [11], Ebert et al. considered a model of microparasite transmission for a horizontally transmitted parasite and established the host-born density-dependent cabin model by ignoring the possibility of host recovery. The model equations are

$$
\left\{\begin{array}{l}
\dot{x}=r(x+f y)[1-c(x+y)]-\mu x-\beta x y, \\
\dot{y}=\beta x y-(\mu+v) y
\end{array}\right.
$$

where $x, y$ are the densities of uninfected (susceptible) and infected (infective) hosts at time $t$, respectively; $r$ is the maximum per capita birth rate of uninfected hosts; $f$ is the relative fecundity of infected hosts; $c$ measures the per capita density-dependent reduction in birth rate; $\mu$ is the parasite-independent host background mortality; $\beta$ is the infection rate constant; and $v$ is the parasite-induced excess death rate.

This model predicts the existence of a stable equilibrium of infected and uninfected hosts, and the population is predicted to approach this equilibrium either monotonically or by damped oscillations.

In this paper, we modify Ebert's model by assuming that infected hosts (bacteria) are capable of reproducing with logistic law, and investigate a class of parasites (phages) infection models. The model is given by the following system of differential equations:

$$
\left\{\begin{array}{l}
\dot{S}=r_{1} S\left(1-\frac{S+I}{M}\right)-d_{1} S-\beta S I, \\
\dot{I}=\beta S I+r_{2} I\left(1-\frac{S+I}{M}\right)-\left(d_{1}+\varepsilon\right) I .
\end{array}\right.
$$

Here, $r_{1}, r_{2}$ are the proliferation constants of uninfected and infected hosts, $M$ is the environmental tolerance of a host population, $d_{1}$ stands for the phage-independent bacteria background mortality, $\beta$ is the proportionality coefficient of parasite infection, $\varepsilon$ denotes the phage-induced excess death rate. In this model, we assume that the total hosts population $N$ is composed of two population classes: the first one consists of uninfected hosts (denoted $S$ ), while the second one consists of the virus infected hosts (denoted $I$ ). Thus, $N(t)=S(t)+I(t)$. We make the following assumption: both uninfected 
and infected hosts are capable of reproducing with logistic law, and the logistic growth of the uninfected bacteria and infected bacteria is given by $r_{1} S(t)[1-(S(t)+I(t)) / M]$ and $r_{2} I(t)[1-(S(t)+I(t)) / M]$, respectively.

To establish our results, the existence and number of steady states, as well as local stability and global stability of uninfected and infected steady states, are analyzed by the Jacobian matrix and Bendixson-Dulac theory. Under certain assumptions (reasonable from the biological viewpoint), we derive the basic reproduction number $R_{0}: E_{1}$ is locally asymptotically stable if $R_{0}<1$, and $E_{1}$ is unstable if $R_{0}>1$. Our theoretical discoveries are supported by numerical simulations.

At present, there is not much work on mathematical modeling of phage infection bacteria, so our study has certain significance. In particular, the parasite (phage) population is not explicitly modeled in this model, which is a striking feature of this model.

The organization of this paper is as follows. In Sect. 2, we discuss the positively invariant set and equilibria. In Sect. 3, we give local and global stability analysis. In Sect. 4, we derive the basic reproduction number. Then, in Sect. 5, we give numerical simulations to support our main result. Section 6 ends the paper with a discussion about phage therapy that could be a new savior for patients infected with superbugs.

\section{Positively invariant set and equilibria}

To begin with, let us find a positively invariant set with respect to (2). Adding the equations in (2), one obtains

$$
\begin{aligned}
\dot{S}+\dot{I} & \leq r_{1} S\left(1-\frac{S+I}{M}\right)+r_{2} I\left(1-\frac{S+I}{M}\right)-d_{1} S-\left(d_{1}+\varepsilon\right) I \\
& \leq r(S+I)\left(1-\frac{S+I}{M}\right)-d_{1}(S+I),
\end{aligned}
$$

where $r=\max \left\{r_{1}, r_{2}\right\}$. Clearly,

$$
\lim _{t \rightarrow \infty} \sup \{S(t)+I(t)\} \leq \bar{N}
$$

where $N=\bar{N}$ is the positive root of the quadratic equation $\left(r-d_{1}\right) N-\frac{r}{M} N^{2}=0$. Hence, the bounded set

$$
\Gamma=\left\{(S, I) \in R_{+}^{2}: S+I \leq \bar{N}\right\}
$$

is positively invariant with respect to (2).

Next, we will study equilibria of system (2) (i.e. zeros of the right-hand side of (2)). It is easy to verify that system (2) admits one vanishing equilibrium and two boundary equilibria. More precisely, the following statement (its proof is straightforward) is true.

Proposition 2.1 Assume that $d_{2}=d_{1}+\varepsilon, 0<d_{i}<r_{i}<1, i=1,2$. Then, system (2) has one vanishing equilibrium $E_{0}=(0,0)$ and two boundary equilibria $E_{1}=(\bar{S}, 0)$ and $E_{2}=(0, \bar{I})$, where $\bar{S}=\frac{M}{r_{1}}\left(r_{1}-d_{1}\right), \bar{I}=\frac{M}{r_{2}}\left(r_{2}-d_{2}\right)$.

Also, the following statement is true. 
Proposition 2.2 Under the assumptions of Proposition 2.1, suppose, in addition, that $\omega:=$ $r_{1} d_{2}-r_{2} d_{1}, \beta_{1}=\max \left\{\frac{r_{2}-r_{1}}{M}, \frac{\omega}{r_{1} \bar{S}}\right\}, \beta_{2}=\min \left\{\frac{r_{2}-r_{1}}{M}, \frac{\omega}{r_{1} \bar{S}}\right\}$. Then system (2) has a unique positive equilibrium $E^{*}=\left(S^{*}, I^{*}\right)$, where

$$
S^{*}=\frac{\omega-\beta r_{2} \bar{I}}{\beta\left(r_{1}-r_{2}+M \beta\right)}, \quad I^{*}=\frac{\beta r_{1} \bar{S}-\omega}{\beta\left(r_{1}-r_{2}+M \beta\right)}
$$

provided that one of the following conditions holds:

(i) $\beta_{1}<\beta<\frac{\omega}{r_{2} \bar{I}}$;

(ii) $\frac{\omega}{r_{2} \bar{I}}<\beta<\beta_{2}$.

Proof Consider the algebraic system

$$
\left\{\begin{array}{l}
r_{1}\left(1-\frac{S+I}{M}\right)-d_{1}-\beta I=0 \\
\beta S+r_{2}\left(1-\frac{S+I}{M}\right)-d_{2}=0
\end{array}\right.
$$

If $r_{1}-r_{2}+M \beta \neq 0$, then (4) admits a unique solution $\left(S^{*}, I^{*}\right)$ given by

$$
\left\{\begin{array}{l}
S^{*}=\frac{M \beta d_{2}-M \beta r_{2}+r_{1} d_{2}-r_{2} d_{1}}{\beta\left(r_{1}-r_{2}+M \beta\right)} \\
I^{*}=\frac{M \beta r_{1}-\alpha M \beta d_{1}-r_{1} d_{2}+r_{2} d_{1}}{\beta\left(r_{1}-r_{2}+M \beta\right)}
\end{array}\right.
$$

Let $\omega:=r_{1} d_{2}-r_{2} d_{1}, \bar{S}:=\frac{M}{r_{1}}\left(r_{1}-d_{1}\right)$ and $\bar{I}:=\frac{M}{r_{2}}\left(r_{2}-d_{2}\right)$. Then (5) is positive if and only if the following condition holds:

$$
\left\{\begin{array}{l}
\left(\omega-\beta r_{2} \bar{I}\right)\left(\beta r_{1} \bar{S}-\omega\right)>0, \\
\left(\omega-\beta r_{2} \bar{I}\right)\left[\left(r_{1}+\beta M\right)-r_{2}\right]>0 .
\end{array}\right.
$$

Since (6) implies $\omega>0$ and $\bar{S}>\bar{I}$, it is easy to see that if $\beta_{1}<\beta<\frac{\omega}{r_{2} \bar{I}}$ or $\frac{\omega}{r_{2} \bar{I}}<\beta<\beta_{2}$, then (2) admits the unique positive equilibrium, and the result follows.

\section{Stability analysis}

\subsection{Local stability of the equilibria $E_{0}, E_{1}, E_{2}$}

We assume that the hypotheses of Propositions 2.1-2.2 are satisfied. The Jacobian matrix of (2) is given by

$$
J(E)=\left[\begin{array}{cc}
r_{1}\left(1-\frac{S+I}{M}\right)-\frac{r_{1} S}{M}-\beta I-d_{1} & -\frac{r_{1} S}{M}-\beta S \\
\beta I-\frac{r_{2} I}{M} & \beta S+r_{2}\left(1-\frac{S+I}{M}\right)-\frac{r_{2} I}{M}-d_{2}
\end{array}\right],
$$

hence,

$$
J\left(E_{0}\right)=\left[\begin{array}{cc}
r_{1}-d_{1} & 0 \\
0 & r_{2}-d_{2}
\end{array}\right]
$$

By assumption, $r_{1}-d_{1}>0$ and $r_{2}-d_{2}>0$, therefore, $E_{0}=(0,0)$ is always an unstable node. 
Next (see (7)):

$$
J\left(E_{1}\right)=\left[\begin{array}{cc}
r_{1}\left(1-\frac{\bar{S}}{M}\right)-\frac{r_{1} \bar{S}}{M}-d_{1} & -\frac{r_{1} \bar{S}}{M}-\beta \bar{S} \\
0 & \beta \bar{S}+r_{2}\left(1-\frac{\bar{S}}{M}\right)-d_{2}
\end{array}\right]
$$

It follows from (9) that the eigenvalues of $J\left(E_{1}\right)$ are

$$
\lambda_{1}:=r_{1}\left(1-\frac{\bar{S}}{M}\right)-\frac{r_{1} \bar{S}}{M}-d_{1}=d_{1}-r_{1} \quad \text { and } \quad \mu_{1}:=\beta \bar{S}+r_{2}\left(1-\frac{\bar{S}}{M}\right)-d_{2}=\frac{\beta r_{1} \bar{S}-\omega}{r_{1}}
$$

By assumption,

$$
d_{1}-r_{1}<0 \text { and } r_{1}>0 \text {, }
$$

hence,

$$
\lambda_{1}<0 \quad \text { and } \quad \operatorname{sign}\left(\mu_{1}\right)=\operatorname{sign}\left(\beta r_{1} \bar{S}-\omega\right)
$$

Finally (see (7)),

$$
J\left(E_{2}\right)=\left[\begin{array}{cc}
r_{1}\left(1-\frac{\bar{I}}{M}\right)-\beta \bar{I}-d_{1} & 0 \\
\beta \bar{I}-\frac{r_{2} \bar{I}}{M} & r_{2}\left(1-\frac{\bar{I}}{M}\right)-\frac{r_{2} \bar{I}}{M}-d_{2}
\end{array}\right]
$$

It follows from (11) that the eigenvalues of $J\left(E_{2}\right)$ are

$$
\lambda_{2}:=r_{2}\left(1-\frac{\bar{I}}{M}\right)-\frac{r_{2} \bar{I}}{M}-d_{2}=d_{2}-r_{2} \quad \text { and } \quad \mu_{2}:=r_{1}\left(1-\frac{\bar{I}}{M}\right)-\beta \bar{I}-d_{1}=\frac{\omega-\beta r_{2} \bar{I}}{r_{2}}
$$

By assumption,

$$
d_{2}-r_{2}<0 \text { and } r_{2}>0 \text {, }
$$

hence,

$$
\lambda_{2}<0 \quad \text { and } \operatorname{sign}\left(\mu_{2}\right)=\operatorname{sign}\left(\omega-\beta r_{2} \bar{I}\right) .
$$

Combining (8)-(12), one obtains the following.

Theorem 3.1 Under the notations and assumptions of Propositions 2.1-2.2, the following statements are true:

(i) $E_{0}$ is always an unstable node;

(ii) If $\beta<\frac{\omega}{r_{1} \bar{S}}$, then $E_{1}=\left(\frac{M}{r_{1}}\left(r_{1}-d_{1}\right), 0\right)$ is locally asymptotically stable; if $\beta>\frac{\omega}{r_{1} \bar{S}}$, then $E_{1}$ is a saddle and, as such, is unstable;

(iii) If $\beta>\frac{\omega}{r_{2} \bar{I}}$, then $E_{2}=\left(0, \frac{M}{r_{2}}\left(r_{2}-d_{2}\right)\right)$ is locally asymptotically stable; if $\beta<\frac{\omega}{r_{2} \bar{I}}$, then $E_{2}$ is a saddle and, as such, is unstable. 


\subsection{Local stability of the positive equilibrium}

It follows from (7) that the Jacobian matrix of (2) at the positive equilibrium point $E^{*}=$ $\left(S^{*}, I^{*}\right)$ is given by

$$
J\left(E^{*}\right)=\left[\begin{array}{cc}
r_{1}\left(1-\frac{S^{*}+I^{*}}{M}\right)-\frac{r_{1} S^{*}}{M}-d_{1}-\beta I^{*} & -\frac{r_{1} S^{*}}{M}-\beta S^{*} \\
\left(\beta-\frac{r_{2}}{M}\right) I^{*} & \beta S^{*}-\frac{r_{2}}{M} I^{*}+r_{2}\left(1-\frac{S^{*}+I^{*}}{M}\right)-d_{2}
\end{array}\right] .
$$

By direct computation,

$$
\begin{aligned}
\operatorname{tr} J\left(E^{*}\right) & =r_{1}\left(1-\frac{S^{*}+I^{*}}{M}\right)-\frac{r_{1} S^{*}}{M}-d_{1}-\beta I^{*}+\beta S^{*}-\frac{r_{2} I^{*}}{M}+r_{2}\left(1-\frac{S^{*}+I^{*}}{M}\right)-d_{2} \\
& =-\frac{r_{1} S^{*}}{M}-\frac{r_{2} I^{*}}{M}<0
\end{aligned}
$$

and

$$
\begin{aligned}
\operatorname{det} J\left(E^{*}\right)= & {\left[r_{1}\left(1-\frac{S^{*}+I^{*}}{M}\right)-\frac{r_{1} S^{*}}{M}-d_{1}-\beta I^{*}\right]\left[\beta S^{*}-\frac{r_{2} I^{*}}{M}+r_{2}\left(1-\frac{S^{*}+I^{*}}{M}\right)-d_{2}\right] } \\
& +\left(\frac{r_{1} S^{*}}{M}+\beta S^{*}\right)\left(\beta-\frac{r_{2}}{M}\right) I^{*} \\
= & \left(\frac{\beta r_{1}}{M}+\beta^{2}-\frac{\beta r_{2}}{M}\right) S^{*} I^{*} \\
= & \frac{\beta}{M}\left[\left(r_{1}+M \beta\right)-r_{2}\right] S^{*} I^{*} .
\end{aligned}
$$

In particular, if $\beta>\frac{r_{2}-r_{1}}{M}$ (resp. $\left.\beta<\frac{r_{2}-r_{1}}{M}\right)$, then $\operatorname{det} J\left(E^{*}\right)>0\left(\right.$ resp. $\left.\operatorname{det} J\left(E^{*}\right)<0\right)$. This way, we arrive at the following result.

Theorem 3.2 Under the notations and assumptions of Propositions 2.1-2.2, the following statements are true:

(i) If $\beta>\frac{r_{2}-r_{1}}{M}$ and $\frac{\omega}{r_{1} \bar{S}}<\beta<\frac{\omega}{r_{2}}$, then $E^{*}$ is locally asymptotically stable;

(ii) If $\beta<\frac{r_{2}-r_{1}}{M}$, then $E^{*}$ is a saddle and, as such, is unstable.

\subsection{Global stability analysis}

We complete this section with the following global stability result.

Theorem 3.3 Under the notations and assumptions of Propositions 2.1-2.2, the following statements are true:

(i) If $\beta<\frac{\omega}{r_{1} \bar{S}}$, then $E_{1}$ is globally asymptotically stable;

(ii) If $\beta>\frac{\omega}{r_{2}}$, then $E_{2}$ is globally asymptotically stable;

(iii) If $\beta>\frac{r_{2}-r_{1}}{M}$, then the unique infection equilibrium $E^{*}$ is globally asymptotically stable.

Proof Take $\mu(S, I)=\frac{1}{S I}$ to serve as a Dulac multiplier.

Denote

$$
P:=r_{1} S\left(1-\frac{S+I}{M}\right)-d_{1} x-\beta S I \quad \text { and } \quad Q:=\beta S I+r_{2} I\left(1-\frac{S+I}{M}\right)-d I .
$$


Then

$$
\frac{\partial(\mu P)}{\partial S}+\frac{\partial(\mu Q)}{\partial I}=-\frac{r_{2}}{M S}-\frac{r_{1}}{M I}<0
$$

for all $S>0, I>0$. Thus, (2) has no periodic orbits in $\Gamma$ (see (3)). A simple application of the classical Poincaré-Bendixson theory shows that all solutions in $\Gamma$ converge to a single equilibrium, from which the result follows.

\section{Basic reproduction number}

In this section, using the method of the next generation matrix (see [21]), we derive the basic reproduction number $R_{0}(\beta)$ of model (2).

We have

$$
F=\beta \bar{S}+r_{2}\left(1-\frac{\bar{S}}{M}\right), \quad V=\left(d_{2}\right),
$$

and the next generation matrix is

$$
F V^{-1}=\frac{1}{d_{2}}\left[\beta \bar{S}+r_{2}\left(1-\frac{\bar{S}}{M}\right)\right] \text {. }
$$

Hence, the basic reproduction number is given by

$$
R_{0}(\beta)=\frac{1}{d_{2}}\left[\beta \bar{S}+r_{2}\left(1-\frac{\bar{S}}{M}\right)\right] .
$$

Clearly (see (13)), $R_{0}(\beta)$ is increasing in $\beta$, and $R_{0}\left(\beta_{c}\right)=1$, where $\beta_{c}=\frac{\omega}{r_{1} \bar{S}}$.

It is easy to see that

$$
\beta \bar{S}+r_{2}\left(1-\frac{\bar{S}}{M}\right)-d_{2}=d_{2}\left(R_{0}(\beta)-1\right) .
$$

Theorem 4.1 Under the notations and assumptions of Propositions 2.1-2.2, the following statements are true:

(i) If $R_{0}(\beta)<1$, then $E_{1}$ is locally asymptotically stable;

(ii) If $R_{0}(\beta)>1$, then $E_{1}$ is a saddle and, as such, unstable.

\section{Numerical simulations}

In this section, we illustrate our results by numeric examples simulating an infection-free equilibrium point and an infection equilibrium point of system (2), as well as give the corresponding time series diagrams. The parameters in the model have been given in Table 1 . In Fig. 1, we choose the death rate $d_{1}=0.01$ day $^{-1}$ and carrying capacity constant $M=10 \mathrm{~mm}^{-3}$. Also, take $\varepsilon=0.2 \mathrm{day}^{-1}, r_{1}=r_{2}=0.4$. Then $\bar{S}=9.75, \bar{I}=4.75, \omega=0.08$, $\frac{\omega}{r_{1} \bar{S}}=0.0205, \frac{\omega}{r_{\bar{I}}}=0.0421$. When $\beta=0.0205$, we have $R_{0}(\beta)=1$.

In Fig. 1(1), we choose $\beta=0.018 \mathrm{~mm}^{3} /$ cells/day, in which case $R_{0}(\beta)<1$. Clearly, the solution approaches the boundary steady state $(\bar{S}, 0)$.

According to the stability results presented in Sect. 3, the above choice of parameter values guarantees that the equilibrium $E_{2}$ is asymptotically stable when $\beta>\frac{\omega}{r_{2} \bar{I}}$. Figure 1(2) shows that, for $\beta=0.052$, the solution approaches the boundary equilibrium $(0, \bar{I})$. 
Table 1 Parameters involved in the model

\begin{tabular}{llll}
\hline Parameter & Parameter describe & Parameter value & Reference \\
\hline$r_{1}$ & proliferation constants of uninfected hosts & 0.4 & {$[11]$} \\
$r_{2}$ & proliferation constants of infected hosts & 0.4 & {$[11]$} \\
$M$ & environmental tolerance of a host population & 10 & assume \\
$d_{1}$ & parasite-independent hosts background mortality & 0.01 & {$[11]$} \\
$\beta$ & humoral response coefficient of a susceptible host population & $0.001-0.1$ & {$[11]$} \\
$\varepsilon$ & parasite-induced excess death rates & 0.2 & {$[11]$} \\
\hline
\end{tabular}
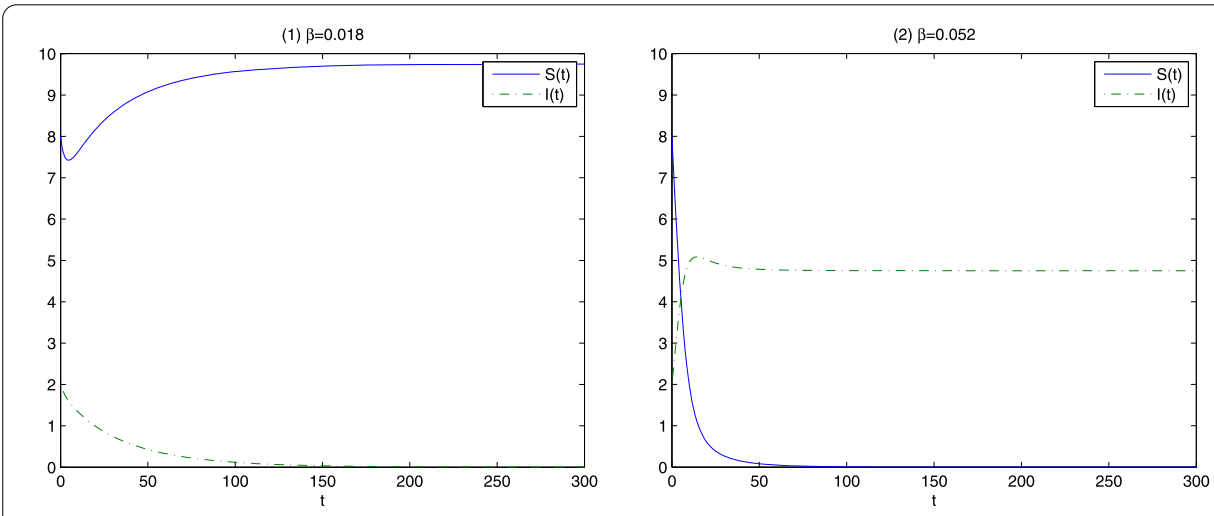

Figure 1 Numerical simulations of system (2) with parameter values $r_{1}=r_{2}=0.4, d_{1}=0.01, \varepsilon=0.2, M=10$

Figure 2 Numerical simulations of system (2) with parameter values $r_{1}=r_{2}=0.4, d_{1}=0.01, \varepsilon=0.2$, $M=10, \beta=0.0039$. Clearly, solution approaches a positive steady state $(0.3879,4.7403)$

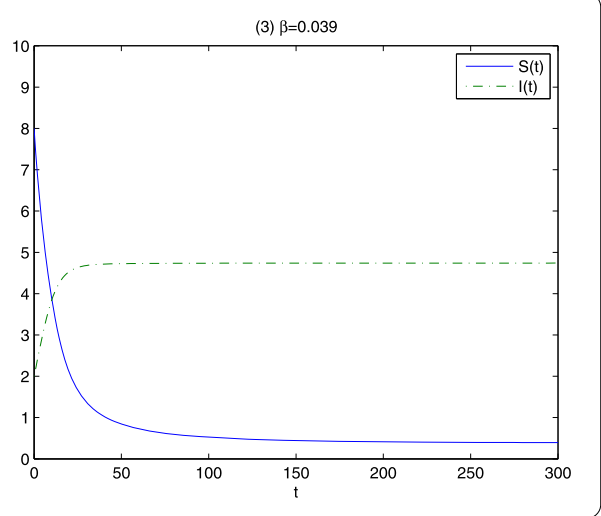

Table 2 Global results for system (2)

\begin{tabular}{lll}
\hline & Conditions & Result \\
\hline 1 & & $E_{0}$ is always an unstable point \\
2 & $\beta<\frac{\omega}{r_{1} \bar{S}}$ & $E_{1}$ is globally asymptotically stable \\
3 & $\beta>\frac{\omega}{r_{2} \bar{l}}$ & $E_{2}$ is globally asymptotically stable \\
4 & $\beta>\frac{r_{2}-r_{1}}{M}, \frac{\omega}{r_{1} \bar{S}}<\beta<\frac{\omega}{r_{2} \bar{l}}$ & $E^{*}$ is globally asymptotically stable \\
\hline
\end{tabular}

Next, taking $\beta=0.039 \mathrm{~mm}^{3} /$ cells/day and leaving other model parameters unchanged, we provide numerical simulations related to the positive equilibrium $E^{*}$ (see Fig. 2). These simulations are consistent with the theoretical results related to the local asymptotic stability of $E^{*}$ obtained in Sect. 3, see Table 2. 


\section{Discussion}

A phage is a virus that lives in bacteria and can infect lysed host bacteria in a specific environment. It has been successfully used to treat infections caused by e. coli, pseudomonas aeruginosa, and staphylococcus (see [22-24]). In recent years, many scholars (both domestic and foreign) conducted in-depth research on phage biology and genome sequencing. It was confirmed that phages of many strains can effectively kill mycobacterium tuberculosis, see $[25,26]$ and the references therein. Also, it was predicted that this "bacterial killer" may become an alternative to antibiotics in the future. There is a complex dynamic relationship between phage and bacteria, which has been described as a variety of dynamic behaviors at the population level, including ARD (arm race dynamics) model [27], FSD (fluctuating selection dynamics) model [27], KtW (kill-the-winner) model [28], and PtW (piggyback-the-winner) model [29]. The study of the dynamic changes of phage and bacteria is helpful to elucidate their changing rules and lay a foundation for the better application of phage in medical treatment, animal husbandry, and other industries. However, there is a lack of research on mathematical modeling of phage infection. Beretta and Kuang [30,31] proposed a mathematical model of phage infection in the ocean and analyzed its mathematical characteristics based on the observations of marine organisms made by biologist A. Okubo. In the present paper, we revised the mathematical model established by Ebert et al. [11] by assuming that (a) infected hosts (bacteria) are capable of reproducing with logistic law, and (b) the relative fecundity of an infected host is equal to zero.

In particular, we studied the nonlinear dynamic model of vertical transmission of infection bacteria. The parasite (phage) population is not explicitly modeled, which is a striking feature of this model. As such, our model is similar to SI-models widely used in epidemiology.

We established four possibilities for the host-parasitic relationships: both uninfected and infected hosts become extinct simultaneously; extinction of uninfected hosts; extinction of infected hosts; uninfected and infected hosts coexist. The local and global stability of the four equilibrium points was analyzed by using the Jacobian matrix and BendixsonDulac theory. In particular, the instability of the extinction equilibrium was established. We also figured out the basic reproduction number $R_{0}$ of the system: $E_{1}$ is locally asymptotically stable (resp. unstable) if $R_{0}<1$ (resp. $R_{0}>1$ ).

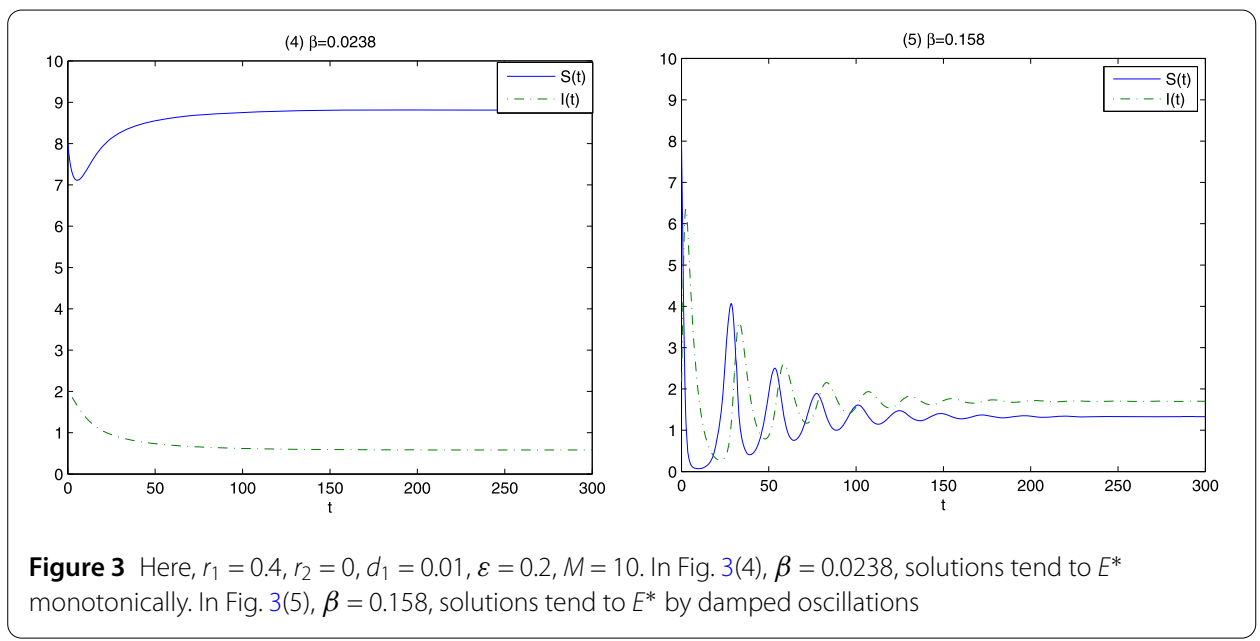


The model simulations presented in Fig. 2 show the existence of the positive equilibrium to which population approaches monotonically. The model simulations presented in Fig. 3 show that when $r_{2}=0$, the population approaches the equilibrium either monotonically or by damped oscillations. Actually, it is a special case of system (1), and the results are consistent with Ebert's prediction in [11]. We established that phages and bacteria can coexist, and the concentration of bacteria can reduce. Also, phages cause the increase in the death rate of the bacterial host, keeping the bacteria at a low concentration that prevents them from becoming ill and spreading to others. Finally, we predict that phage therapy could be a new savior for patients infected with superbugs.

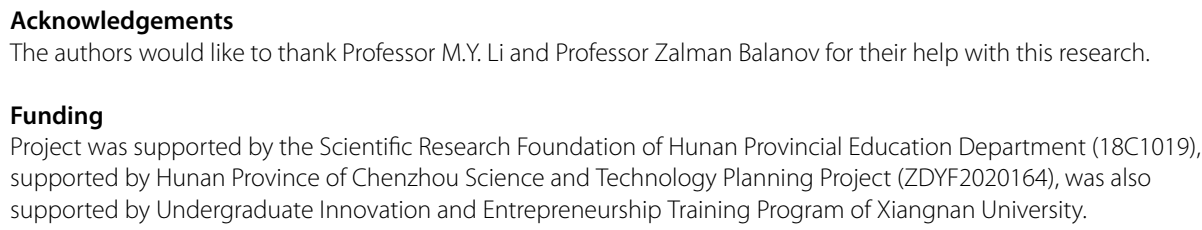

\section{Authors' information}

Xiaoping Li, Associate professor, her main research field is fractional differential equations and boundary value problems. Rong Huang, Associate professor, her main research field is fractional differential equations and boundary value problems. Minyuan He, Lecturer, her main research field is probabilistic statistics.

\section{Author details}

${ }^{1}$ College of Mathematics and Finance, Xiangnan University, Hunan, P.R. China. ${ }^{2}$ Department of Mathematical Sciences, Tianjin Normal University, Tianjin, P.R. China.

\section{Publisher's Note}

Springer Nature remains neutral with regard to jurisdictional claims in published maps and institutional affiliations.

Received: 20 October 2020 Accepted: 26 April 2021 Published online: 08 November 2021

\section{References}

1. Twort, F.W:: An investigation on the nature of ultra-microscopic viruses. Lancet 186(4814), 1241-1243 (1915)

2. D'Herelle, F.: Sur un microbe invisible antagoniste des bacilles dysenteriques [An invisible microbe that is antagonistic to the dysentery bacillus]. C. R. Acad. Sci. 165, 373-375 (1917) (in French)

3. Gallaway, T.R., et al.: Evaluation of phage treatment as a strategy to reduce salmonella populations in growing swine. Foodborne Pathog. Dis. 8(2), 261-266 (2011)

4. Cha, S.B., et al.: Effect of bacteriophage in enterotoxigenic Escherichia coli (ETEC) infected pigs. J. Vet. Med. Sci. 74(8), 1037-1039 (2012)

5. Stalin, N., Srinivasan, P.: Efficacy of potential phage cocktails against Vibrio harveyi and closely related Vibrio species isolated from shrimp aquaculture environment in the South East coast of India. Vet. Microbiol. 207, 83-96 (2017)

6. Woolston, J., et al.: Bacteriophages lytic for Salmonella rapidly reduce Salmonella contamination on glass and stainless steel surfaces. Bacteriophage 3(3), e25697 (2013)

7. Nkwe, K.I., Ateba, C.N., Sithebe, N.P., et al.: Enumeration of somatic and F-RNA phages as an indicator of fecal contamination in potable water from rural areas of the North West Province. Pathogens 4(3), 503-512 (2015)

8. Wu, B., Wang, R., Fane, A.G.: The roles of bacteriophages in membrane based water and wastewater treatment processes: a review. Water Res. 110, 120-132 (2017)

9. Perlson, A.S., Nelson, P.W.: Mathematical analysis of HIV-I dynamics in vivo. SIAM Rev. 41, 3-14 (1999)

10. Nowak, M.A., May, R.M.: Virus Dynamics. Oxford University Press, New York (2000)

11. Ebert, D., Lipsitch, M., Mangin, K.T.: The effect of parasites on host population density and extinction: experimental epidemiology with Daphnia and six microparasites. Am. Nat. 156, 459-477 (2000)

12. Regoes, R.R., Ebert, D., Bonhoeffer, A.: Dose-dependent infection rates of parasites produce the Allee effect in epidemiology. Proc. R. Soc. Lond. B 269, 271-279 (2002)

13. Hwang, T.W., Kuang, Y.: Deterministic extinction effect of parasites on host populations. J. Math. Biol. 46, 17-30 (2003) 
14. Gomez-Acevedo, H., Li, M.Y.: Backward bifurcation in a model for HTLV-l infection of CD4 ${ }^{+}$T cells. Bull. Math. Biol. 67 101-114 (2005)

15. Wang, L., Li, M.Y.: Mathematical analysis of the global dynamics of a model for HIV infection of CD4 ${ }^{+}$T cells. Math. Biosci. 200, 44-57 (2006)

16. Hasan, B., Devendra, K., et al.: Analytic study for a fractional model of HIV infection of CD4+ T lymphocyte cells. Math. Nat. Sci. 2, 33-43 (2018)

17. Li, M.Y., Wang, L.: Backward bifurcation in a mathematical model for HIV infection in vivo with anti-retroviral treatment. Nonlinear Anal., Real World Appl. 17, 147-160 (2014)

18. Zhou, Y., Yang, Y., Zhang, H.: Stability of non-monotone critical waves in a population dynamics model with spatio-temporal delay. Math. Nat. Sci. 2, 8-23 (2018)

19. Abdon, A., Seda, I.: Mathematical model of COVID-19 spread in Turkey and South Africa: theory, methods and applications. Adv. Differ. Equ. 2020, 659 (2020)

20. Abdon, A., Seda, I.: Nonlinear equations with global differential and integral operators: existence, uniqueness with application to epidemiology. Results Phys. 20, 103593 (2021)

21. van den Driessche, P., Watmough, J.: Reproduction numbers and sub-threshold endemic equilibria for compartmental models of disease transmission. Math. Biosci. 180, 29-48 (2002)

22. McNerney, R., Traoré, H.: Mycobacteriophange and their application to disease control. J. Appl. Microbiol. 99(2), 223-233 (2005)

23. Rybniker, J., Kramme, S., Small, P.L.: Host range of 14 mycobacteriophanges in mycobacterium ulcerans and seven other mycobacteria including Mycobacterium tuberculosis-application for identification and susceptibility testing. J. Med. Microbiol. 55(Pt 1), 37-42 (2006)

24. O'Flaherty, S., Ross, R.P., Coffey, A.: Bacteriophage and their lysins for elimination of infectious bacteria. FEMS Microbiol. Rev. 33(4), 801-819 (2009)

25. Pasechnik, V.A., Roberts, A.D.G., Sharp, R.J.: Treatment of intracellular infection. US 6660264 [P], 2003-12-09

26. Jones, W.D., Good, R.C., Thompson, N.J., et al.: Bacteriophage types of Mycobacterium tuberculosis in the United States. Am. Rev. Respir. Dis. 125(6), 640-643 (1982)

27. Mirzaei, M.K., Maurice, C.F.: Menage a trois in the human gut: interactions between host, bacteria and phages. Nat. Rev. Microbiol. 15, 397-408 (2017)

28. Lim, E.S., Zhou, Y., Zhao, G., et al.: Early life dynamics of the human gut virome and bacterial microbiome in infants. Nat. Med. 12, 1228-1234 (2015)

29. Shkoporov, A.N., Hill, C.: Bacteriophages of the human gut: the "known unknown" of the microbiome. Cell Host Microbe 25, 195-209 (2019)

30. Beretta, E., Kuang, Y.: Modeling and analysis of a marine bacteriophage infection. Math. Biosci. 149, 57-76 (1998)

31. Beretta, E., Kuang, Y.: Modeling and marine bacteriophage infection with latency period. Nonlinear Anal., Real World Appl. 2, 35-74 (2001)

\section{Submit your manuscript to a SpringerOpen ${ }^{\circ}$ journal and benefit from:}

- Convenient online submission

- Rigorous peer review

- Open access: articles freely available online

- High visibility within the field

- Retaining the copyright to your article

Submit your next manuscript at $>$ springeropen.com 